\title{
Erratum: Clinical Profiles, Characteristics, and Outcomes of the First 100 Admitted COVID-19 Patients in Pakistan: A Single- Center Retrospective Study in a Tertiary Care Hospital of Karachi
}

Muhammad Sohaib Asghar ${ }^{1}$, Syed Jawad Haider Kazmi ${ }^{2}$, Noman Ahmed Khan ${ }^{3}$, Mohammed Akram $^{4}$, Salman Ahmed Khan ${ }^{5}$, Uzma Rasheed ${ }^{4}$, Maira Hassan ${ }^{4}$, Gul Muhammad Memon ${ }^{4}$

1. Internal Medicine, Dow International Medical College, Dow University Hospital, Dow University of Health Sciences, Karachi, PAK 2. Emergency Medicine, Liaquat National Hospital and Medical College, Karachi, PAK 3. General Surgery, Liaquat National Hospital and Medical College, Karachi, PAK 4. Internal Medicine, Liaquat National Hospital and Medical College, Karachi, PAK 5. Internal Medicine, Dow International Medical College, Karachi, PAK

Corresponding author: Muhammad Sohaib Asghar, sohaib_asghar123@yahoo.com

Corresponding author: Muhammad Sohaib Asghar

1. Internal Medicine, Dow International Medical College, Dow University Hospital, Dow University of Health Sciences, Karachi, PAK 2. Emergency Medicine, Liaquat National Hospital and Medical College, Karachi, PAK 3. General Surgery, Liaquat National Hospital and Medical College, Karachi, PAK 4. Internal Medicine, Liaquat National Hospital and Medical College, Karachi, PAK 5. Internal Medicine, Dow International Medical College, Karachi, PAK 6. Internal Medicine, Liaquat National Hospital and Medical College, Karachi, PAK 7. Internal Medicine, Liaquat National Hospital and Medical College, Karachi, PAK 8. Internal Medicine, Liaquat National Hospital and Medical College, Karachi, PAK

How to cite this erratum

Asghar M, Haider Kazmi S, Ahmed Khan N, et al. (August 06, 2020) Correction: Clinical Profiles, Characteristics, and Outcomes of the First 100 Admitted COVID-19 Patients in Pakistan: A Single-Center Retrospective Study in a Tertiary Care Hospital of Karachi. Cureus 12(8): c34. doi:10.7759/cureus.c34

\section{Correction Notice}

Tables 3 and 5 included the following incorrect data. These data points have been corrected.

- Table 3: In the serum urea section, the mean in ICU stay is $58.33 \pm 37.22$. The standard deviation was originally incorrectly entered as 1.94 by the authors and has been replaced with 37.22.

- Table 5: In the basophil section, the cut off in death is 0.50 . The value was originally incorrectly entered as 655.00 by the authors and has been replaced with 0.50 . 